\title{
The Role of Helicobacter pylori Infection, and Malnutrition among Type 2 Diabetic Medical Services Patients in the Gaza Strip
}

\author{
Mazen A. El-Sakka ${ }^{1}$, Ektemal M. Abu Jabal' ${ }^{2}$, Luay M. Nasser ${ }^{3}$ \\ ${ }^{1}$ Pharmacognosy Department, Faculty of Pharmacy, Al Azhar University-Gaza, Gaza, Palestine; ${ }^{2}$ Nutrition Department, Ministry of \\ National Economy, Gaza, Palestine; ${ }^{3}$ Medical Service, Gaza, Palestine. \\ Email: sakkamazen@gmail.com
}

Received October $12^{\text {th }}, 2013$; revised November $10^{\text {th }}$, 2013; accepted December $3^{\text {rd }}, 2013$

Copyright (C) 2013 Mazen A. El-Sakka et al. This is an open access article distributed under the Creative Commons Attribution License, which permits unrestricted use, distribution, and reproduction in any medium, provided the original work is properly cited.

\begin{abstract}
Background: Diabetes means the blood glucose, which is too high or too low. With Type 2 DM, the more common type, the body does not make or use insulin well. In patients with DM, Helicobacter pylori is one of the most common infections worldwide. Available data on the possible association between H. pylori infection and DM are contradictory. There are a few studies in the Middle East, and this study is the pioneer study, in the Medical Services Clinics in Gaza strip. Aims: This study was conducted to reveal the prevalence of $H$. pylori infection, malnutrition, insulin resistance among T2DM patients, to describe the dietary requirements of T2DM patients, finally to evaluate the current information about diet, and lifestyle in the prevention of $\mathrm{H}$. pylori, and malnutrition. Methodology: A cross-sectional study was conducted in the Medical Services Clinics in Gaza Strip, and there were 129 patients included in this study. Data were collected through hematological information and structured interview questionnaire. Results: Highly significant percentage of $\mathrm{H}$. pylori (70\%) among the DM patients includes in the study, but not indicates any significant association between gender and $H$. pylori status. Conclusion: $H$. pylori patients should update their sugar level values in the record, and should get exercise and diet plan for every meal.
\end{abstract}

Keywords: Type 2 Diabetes Mellitus (T2DM); Helicobacter pylori (H. pylori); Insulin Resistance (IR); Malnutrition

\section{Introduction}

In recent years, a significant association has been reported between cardiovascular diseases, diabetes mellitus, and dyslipidemia and Helicobacter pylori (H. pylori) infection. However, there are conflicting reports, and lack of data from the Middle East should prompt local studies for establishing the relationship between $H$. pylori and such chronic diseases [1].

In patients with diabetes mellitus (DM), chronic infections are frequent and severe, due to the impairment of their immune status. However, data on the prevalence of Helicobacter pylori infection in diabetics are scanty and contradictory. Patients with insulin-dependent diabetes mellitus (IDDM) are often affected by chronic infections [1].

Diabetes Mellitus means the blood glucose, or blood sugar are too high. With Type 2 diabetes mellitus, the more common type, the body does not make or use insulin well. Insulin is a hormone that helps glucose gets into the cells to give them energy. Without insulin, too much glucose stays in the blood. Over time, high blood glucose can lead to serious problems with heart, eyes, kidneys, nerves, gums and teeth [2].

Helicobacter pylori are one of the most common infections worldwide. Available data on the possible association between $H$. pylori infection and diabetes mellitus (DM) are contradictory [3].

That is clearly indicated there is a considerable nutritional public problem. The intention of the study is to assess the nutritional status of the diabetic patients, whom have $H$. pylori bacteria in their stomach, and to determine the nutritional factors contributing in development of nutritional diabetic patients [4].

Since there are only a few studies in the Middle East, on the association of Helicobacter pylori and diabetes mellitus, we conducted this study and focus on the frequency of Helicobacter pylori infection, Insulin resis- 
tance and malnutrition, in patients with type 2 diabetes mellitus, and help in providing data that is useful in the field of medicine as well as epidemiology.

\subsection{The Relationship between T2DM and H. pylori}

Many authors have extensively explored the relationship between $H$. pylori and diabetes mellitus.

Robert P study in 2012 show there may be a link between $H$. pylori bacteria and type 2 diabetes mellitus in adults, the researchers analyzed data from people who took part in two US National Health and Nutrition Surveys and found that the presence of $H$. pylori bacteria was consistently associated with elevated levels of glycosylated hemoglobin (HbA1c), an indicator of blood glucose levels and diabetes mellitus, the association was strongest in obese people [5].

According to Albaker study from Saudi Arabia, it shows that the association of Metabolic Syndrome (MS) and $H$. pylori is still controversial with emphasis on the possible linkage between them. However, the high prevalence of both MS and $H$. pylori infection might explain the coincidence [6].

Ramazan Gen in 2010, studied the relationship between Helicobacter pylori infection and insulin resistance, in a prospective, open-label, single-center study which consisted of 159 patients. The study showed beneficial effects of $H$. pylori eradication on insulin resistance, atherogenic lipid abnormalities and low-grade inflammation. The results suggest that $H$. pylori eradication may prevent coronary artery disease and metabolic syndrome [7].

\section{Subjects and Methods}

\subsection{Study Design}

The current cross sectional study was conducted to determine the prevalence of $H$. pylori infection in type 2 DM patients.

\subsection{Study Setting}

This study was carried out in the Medical Services Clinics in the Gaza Strip [8].

\subsection{Target Population}

The study population consisted of 138 diabetic patient's type 2, male \& female patients' presents in Medical Services clinics in the Gaza Strip, the sample number was calculated by Decision Analyst State 2.0 programs.

\subsection{Study Period}

The proposed study was conducted from October 2011 to
May 2012.

\subsection{Sampling}

A Cross-Sectional Study was done randomly selected on 129 Diabetic patients with Type 2, from the Medical Services Patients in the Gaza Strip.

\subsection{Data Collection \& Health Records}

Health records of each type 2 diabetic patients were reviewed through the Medical Services Clinics in the Gaza strip conform A, B, C and D methods.

1) Anthropometric Measurements

- Weight in kg measured by a seca scale

- Height in $\mathrm{cm}$ measured by a stadiometer

- Body Mass Index (BMI)

- Waist circumference

\section{2) Biochemical Assessment}

- FBS

- HbA1c

- C-Peptide

- H. pylori (IgG)

3) Clinical findings

It includes a complete physical examination, to investigate signs of malnutrition, medical history e.g. including acute and chronic illness, diagnostic procedures, therapies, treatments that may increase nutrient needs or induce malabsorption, current medications.

\section{4) Dietary assessment}

It involves estimating food intake. This can be achieved by interview techniques (usually retrospective) and recording of intake.

\section{Results}

The frequency and percentage of $H$. pylori are given in Table 1, and around $70.5 \%$ of the sample found has positive H. pylori.

\subsection{Correlation between Gender and $H$. pylori}

It is found there is no significant difference between the means of chemical variables due to the gender, and there is insignificant association between $H$. pylori and the gender as seen in Table 2.

Table 1. Distribution of $\boldsymbol{H}$. pylori.

\begin{tabular}{cc}
\hline H. pylori & No. (\%) \\
\hline Positive & $91(70.5 \%)$ \\
Negative & $38(29.5 \%)$ \\
Total & $129(100.0 \%)$ \\
\hline
\end{tabular}


Table 2. Test of association between gender and $H$. pylori.

\begin{tabular}{|c|c|c|c|c|c|}
\hline \multirow{2}{*}{\multicolumn{2}{|c|}{ H. pylori }} & \multicolumn{2}{|c|}{ Gender } & \multirow{2}{*}{ Chi square } & \multirow{2}{*}{ P-value } \\
\hline & & Male & Female & & \\
\hline Positive & No. (\%) & 49 (53.9\%) & 42 (46.1\%) & \multirow{2}{*}{1.478} & \multirow{2}{*}{0.224} \\
\hline Negative & No. (\%) & 16 (42.1\%) & 22 (57.9\%) & & \\
\hline
\end{tabular}

\subsection{Diabetes History}

Results in Table 3 shows that there are significant differences between the means of the C-peptide and HbA1c categories at 0.05 level of significant where there is a significant difference between the means of the FBS categories at 0.1 level of significant.

\subsection{Physical Activity}

The results showed that the most popular physical activity of sample practice is walking (46.51\%) of sample practice it, followed by house work (38.76\%). The present study observed a statistically significant association between the means of the C-peptide for different activity classes. On the other hand, almost half of the samples sleep less than 8 hours, where the other half of the samples sleeps more than 8 hours.

\subsection{Dietary Assessment}

The results of this study highlights Grains, starches, bran and mixed bread, fruit and vegetables, desserts, animal fat, fried food, olive oil, fish, vegetables, fruits, proteins, legumes, nuts, cold drinks, soda beverages and hot drinks, tea, coffee, and nuts which considered as the main dietary factors that may associated positively or negatively with type $2 \mathrm{DM}$.

\section{Discussion}

\subsection{Distribution of $\boldsymbol{H}$. pylori}

The results of this study showed a highly significant percentage of $\mathrm{H}$. pylori (70.5\%) among the diabetic patients including in the study, this result is consistent with some literature and inconsistent with others.

The results are supported by the study of Bener, and Simon's their results found that Helicobacter pylori infection was significantly higher in diabetic patients than non-diabetic subjects $[9,10]$.

\subsection{Correlation between Gender and Chemical Variables}

The current study revealed no significant association between the means of chemical variables (FBS, C-peptide, and $\mathrm{HbA} 1 \mathrm{c}$ ) according to the gender.
Table 3. The chemical variables based on the diabetes mellitus history.

\begin{tabular}{|c|c|c|c|c|c|c|c|}
\hline \multirow{2}{*}{$\begin{array}{l}\text { How long } \\
\text { have you } \\
\text { diabetes? }\end{array}$} & \multicolumn{3}{|c|}{ FBS } & \multicolumn{2}{|c|}{ C-peptide } & \multicolumn{2}{|c|}{ HbA1c } \\
\hline & $\mathrm{N}$ & Mean & SD & Mean & SD & Mean & SD \\
\hline $\begin{array}{l}\text { Less than or } \\
1 \text { year }\end{array}$ & 25 & 127.64 & 58.19 & 1.01 & 0.20 & 6.26 & 1.14 \\
\hline 1 - 5 years & 32 & 148.06 & 56.37 & 1.70 & 0.30 & 6.53 & 1.0 \\
\hline 5 - 10 years & 26 & 131.23 & 25.81 & 1.08 & 0.21 & 6.53 & 1.14 \\
\hline $\begin{array}{l}\text { More than } \\
10 \text { years }\end{array}$ & 46 & 154.35 & 41.03 & 0.88 & 0.13 & 7.40 & 1.19 \\
\hline Total & 129 & 142.95 & 47.47 & 1.23 & 0.11 & 6.79 & 1.21 \\
\hline F-value & & \multicolumn{2}{|c|}{2.487} & \multicolumn{2}{|c|}{3.554} & \multicolumn{2}{|c|}{7.408} \\
\hline P-value & & \multicolumn{2}{|c|}{$0.064^{* *}$} & \multicolumn{2}{|c|}{$0.016^{*}$} & \multicolumn{2}{|c|}{$0.000^{*}$} \\
\hline
\end{tabular}

*Significant at 0.05 level of significant. ${ }^{* *}$ Significant at 0.1 level of significant.

No statistically significant difference found between the socioeconomic variables according to the gender. This does not indicate any significant association between gender and $H$. pylori status.

\subsection{Diabetes History}

This study identifies significant differences between the presence of $H$. pylori and diabetes mellitus history.

This result similar to Robert $\mathrm{P}$ study, the findings show there may be a link between $H$. pylori bacteria and type 2 diabetes mellitus in adults, and found that the presence of $H$. pylori bacteria was consistently associated with elevated levels of glycosylated hemoglobin (HbA1c) [5].

\subsection{Dietary Habits}

In general the current study shows that there are insignificant correlation between food groups and Chemical variables, but there are significant correlation between vegetables and C-peptide, legumes and C-peptide. And there is a statistically significant correlation detected between vegetables, hot drink and HbA1c.

The results also reported that there is no significant difference between the means of the food groups with respect to the $H$. pylori status.

\subsection{Grains, Starches}

The result of the present study showed that slightly more than two thirds (84.5\%) of patients which is the high percentage of white breads intake of grains, starches once or more weekly, $15 \%$ eating saj breads once or more weekly, and more than a half of patients about $68.2 \%$ didn't eat brown breads with bran at all, and 56.6\% 
didn't eat brown breads without bran at all. The present study results show that $11.6 \%$ from the samples eating macaroni/spaghetti once per day or more, and $47.3 \%$ of patient didn't intake it at all.

In general terms, most of the studies revealed that consumption of refined sugars is directly associated with diabetes mellitus disease [11].

\subsection{Fruit}

The current study shows that more than two thirds (79.1\%) of patients eating and drinking citrus as orange grapefruits and lemons, once per day or more, and only $1.6 \%$ percentage didn't intake it at all.

The results show that $60.5 \%$ of patients eating the apples once or more per day, then $34.9 \%$ patients eaten watermelon once or more per day, in the end of the fruits list comes the pineapple (fresh/canned) which taken by $17.8 \%$ of the diabetic patients.

\subsection{Vegetables}

Some studied shows that the regular consumption of fruit and vegetables is associated with reduced risks of chronic diseases, where they contain significant amounts of bioactive components and fibers that may provide desirable health benefits beyond basic nutrition [12]. The current study shows that (34\% - 44\%) of patients eating a cooked vegetables as okra, molokhia, zucchini, potato (cooked/boiled/or mashed), green beans, spinach, once or more per day, and the results shows that about $67.4 \%$ from patients are eating vegetable salad daily, and in other patients taken once or more per day.

\subsection{Proteins}

The study results show that more than $40 \%$ from diabetic patient intake milks, yogurts, cheeses, eggs, and between $18 \%$ - 30\% from the samples didn't eat them at all.

And about $69.8 \%$ from chickens, that's because the diabetic patients including in the study prefer the chicken more than other meats and intake it once or more per weeks, where $1.6 \%$ from patients don't eat it at all.

\subsection{Legumes}

The results show that diabetic patients often eating legume as lentil, pea, chickpeas, bean with sesame paste, green beans, about $50 \%$ - $55 \%$ of patients eating legumes one or more per day, and $10 \%$ from patients didn't eat legumes at all.

\subsection{Nuts}

The results show that $20 \%$ - $24 \%$ from patients eats nuts as (peanuts, walnuts, sunflower, watermelon, almonds), once or more per weeks, and the higher percentage of patients didn't eat it at all.

\subsection{Desert}

The results show that $11.6 \%$ only from the diabetic patients eat keck (others), and 30.6\% from samples eat home sweats, but the higher percentage of diabetic patients didn't eats keck or oriented sweats at all, because of their effects of elevated the blood sugar.

\subsection{Cold \& Hot Drinks}

The results found that $57.4 \%$ drinks the fresh fruits juice, and $31 \%$ from patients didn't drink it at all, about $21.7 \%$ drink soda, and $57.4 \%$ from the samples didn't drinks it at all, about $76.7 \%$ from patients drinks tea, $55 \%$ drinks coffee, and $20 \%-24 \%$ from them drinking cinnamon, ginger, and herbal tea.

\subsection{Fried Food}

The study showed consumption of fried foods and excessive oil about $52.7 \%$ eats (flafel, potato, eggplants, cauliflower), and $18.6 \%$ only didn't eat it at all.

\subsection{Fast Food}

The results showed little consumption of fast foods (as pizza, shawerma, borger, thailandy) only about 3.9\% 9.3\% eats fast foods, and 85\% didn’t eat it at all.

\subsection{Spices}

The results found that all the samples takes spices as red/green pepper black pepper, curcuma, other spices with in the foods, $79.8 \%$ - $82 \%$ eat it once or more per day.

\subsection{Pickles}

The results show that $43.4 \%$ intake the pickles as homemade olives once or more per day, and 37.2\% didn't eat the homemade, and $70.0 \%$ from the patient didn't eat any pickles from market at all.

\section{Conclusions \& Recommendation}

In the present study, $H$. pylori infection, insulin resistance and malnutrition in type 2 DM patients, were done for all subjects.

1) An adequate lifestyle, socioeconomic factors, and some biochemical alterations could play an important role in the etiology of type $2 \mathrm{DM}$ patients.

2) This study demonstrates the strong relationship of $H$. pylori infection as a risk factor for insulin resistance, more than $70.5 \%$ of the samples found to have positive $H$. py- 
lori.

3) Greater attention of the eradication of $H$. pylori has been shown to play important roles in the etiology of other chronic diseases.

4) We can conclude that there is insignificant association between the $H$. pylori and diabetes mellitus history (the duration of diabetes). H. pylori infection is not associated with duration of diabetes,

5) The study exhibited a positive significant difference between the means of weight for persons with positive and negative $H$. pylori status. Consequently, the mean of BMI of positive groups is significantly higher than the mean BMI for the negative group.

6) The results suggest that data seem to indicate a potential association between $H$. pylori infection, malnutrition and IR, and further studies are needed to strengthen this association and to clarify whether there is a causative link between them.

Serum $H$. pylori should be integrated into routinely para-clinical investigations in all patients with type 2 DM.

\section{REFERENCES}

[1] A. Baradaran and H. Nasri, "Helicobacter pylori Specific IgG Antibody and Serum Magnesium in Type-2 Diabetes Mellitus Chronic Kidney Disease Patients,” Saudi Journal of Kidney Diseases and Transplantation, Vol. 22, No. 2, 2011, pp. 282-285.

[2] National Institutes of Health (NIH), "A Service of the U.S. National Library of Medicine. Annual Report,” 2012,

[3] S. A. Polyzos, J. Kountouras, C. Zavos and G. Deretzi, "The Association between Helicobacter pylori Infection and Insulin Resistance; Part 1,” Heicobacter Journal, Vol. 16, No. 1, 2010, pp. 76-88.
[4] M. D. Goutham Rao, "Insulin Resistance Syndrome," American Family Physician, Vol. 63, No. 6, 2001, pp. 1159-1164.

[5] R. Preidt, “Type of Bacteria May Be Linked to Diabetes, The Association between $H$. pylori Infection, Type 2 Disease,” A Service of the US National Library of Medicine, 2012.

[6] W. I. Albaker, "Helicobacter pylori Infection and Its Relationship to Metabolic Syndrome: Is It a Myth or Fact?” Saudi Journal of Gastroenterology, Vol. 17, No. 3, 2011, pp. 165-169. http://dx.doi.org/10.4103/1319-3767.80377

[7] R. Gen, M. Demir and H. Ataseven, "Effect of Helicobacter pylori Eradication on Insulin Resistance, Serum Lipids and Low-Grade Inflammation,” Southern Medical Journal, Vol. 103, No. 3, 2010, pp. 190-196. http://dx.doi.org/10.1097/SMJ.0b013e3181cf373f

[8] Medical Services Commission, Annual Report 2010/ 2011.

[9] A. Bener, S. A. Uduman, A. Ameen, et al., "Prevalence of Helicobacter pylori Infection among Low Socio-Economic Workers," Journal of Communicable Diseases, Vol. 34, No. 3, 2002, pp. 179-184.

[10] L. Simon's, J. Tornoczky, M. Toth, M. Jambor and Z. Sudar, "The Significance of Campylobacter pylori Infection in Gastroenterologic and Diabetic Practice,” Orvosi Hetilap, Vol. 130, No. 25, 1989, pp. 1325-1329.

[11] A. Cuevas, J. Miquel, M. Reyes and F. Nervi, "Diet as a Risk Factor for Cholesterol Gallstone Disease,” Journal of the American College of Nutrition, Vol. 23, No. 3, 2004, pp. 187-196.

[12] R. Liu, "Health Benefits of Fruit and Vegetables Are from Additive and Synergistic Combinations of Phytochemicals," American Journal of Clinical Nutrition, Vol. 78, No. 3, 2003, pp. 517-520. 